\title{
Christophe Girot
}

\section{Cloudism}

A quiet cloudist revolution has taken place in the culture of making landscape architecture. This has come with a profound conceptual shift in aesthetic representation brought about by new digital tools and methods pertaining to landscape analysis and design. Projects are now conceived digitally as physical entities in the full topological sense of the word. Various aspects of a terrain can be worked at as a body and put into relationship to one another. The environment is changing rapidly and requires a different approach to problem solving and to the material culture and fabrication of landscapes. We are currently being asked to change the shape of natural things through a mix of science and artistry. The fact that nature is understood now as a rapidly evolving global phenomenon has marked our awareness of the world with a sense of imminent urgency, one that calls for an immediate response with tools of a different kind. The tools must be performative and transdisciplinary to accommodate new operational dimensions in design. ${ }^{1}$ Cloudism is the term invented to describe the new art of thinking and making landscape architecture using point cloud modelling as base. Designers adopting cloudism will step into an overwhelmingly convincing simulacrum of physical reality, space and time, this will enhance their understanding of a site, and yield a stronger awareness of ambient aspects and queues.

\section{Building a Cloud}

Point cloud technology finds its roots in the early development of laser scanners and their application to satellite reconnaissance and terrestrial mapping. Lidar is an acronym for 'light and radar' used both for airborne surveys and mobile terrestrial scans. It was developed in the 1960s at the US National Centre for Atmospheric Research for applications in meteorology and by NASA as an altimeter to map the surface of the moon in the Apollo 15 mission of the early 1970s. The Experimental Cartographic Unit (ECU) at Cavendish Laboratory in Cambridge, England, pioneered a first prototype of a laser scanner for particle research, called the 'Sweepnik', in the 1960 s. $^{2}$ As mainframe computers and lasers evolved, so did landscape feature detection and measurement techniques in the environment. ${ }^{3}$ Both the precision and range of laser scanning devices have improved, as have their potential applications. 
Terrestrial Laser Scanners (TLS) function either with a narrow infrared or ultraviolet ray and are able to map distant features at a very high resolution with hundreds of points per square metre. Several publications heralded the birth of this new technique decades ago. The term point cloud for instance was first coined in the mid 1980s by a team of researchers at Chapel Hill. ${ }^{4}$ But it is really at the turn of the millennium that cloud computing began to develop and spread more widely in the architectural and engineering fields with the rapid digitalisation of ground breaking surveying and modelling techniques. Currently, point cloud data sets can be sampled either with Lidar or TLS technologies. Every device produces a spherical pixel cloud of information around a recording point. The device maps all visible features within a given range from the source. It is ideal for landscapes where data is not easily available, but it is also suited to complex urban projects where more specific site data may be required. Point cloud models allow various data sets with varying point densities to be combined. The different data sets all align within a three-dimensional coordinate system which positions them precisely relative to each other in geographic space. The laser scanner operates by measuring the distance to the first object on its path and returns the information to a sensor at the speed of light, giving a precise reading of the height, depth and position of the pixel received. It is with this incredible degree of precision that an entire landscape can be apprehended and recorded.

On-site immersion while scanning is paramount to garnering unique field observations that become an integral part of the feeling for a place. The follow-up in studio through different stages of design enables a better comprehension of the environment, as well as physical and spatial conditions at stake. The point cloud model also enables the discretionary development of design solutions that can be repeatedly tested and adapted to a broader context. Any part of a point cloud model can be subdivided, extracted and developed locally to test specific design implementations in the physical realm. The extracted piece can then be fitted back into the overall site model and integrated into a larger system for simulation and evaluation purposes. In the cloudist approach, there exists no separation between a model, a section and a plan, they all stem from the same cloud of design information. In the case of cloudism, separate renderings and visualisations become quite unnecessary since the views generated are directly derived from the model, with their own singular aesthetic.

\section{Field Experiments}

The cloudist research and development at the Landscape Visualizing and Modelling Laboratory (LVML) of ETH has succeeded in mastering the modelling of large scale landscapes, using point cloud data sets in their entirety (100\%). ${ }^{5}$ The geographically positioned models can be used for various applications in landscape design, analysis and simulation. Each model can comprise up to 1 billion points of information. Research and teaching has shifted from conventional contour modelling and GIS overlay mapping, towards more dynamic and versatile forms of landscape exploration within the cloud.

The Swiss Cooperation Project started back in 2010 under the leadership of 
Fig. 1 (below) Section Gotthard Motorway intersection in Airolo Ticino, Switzerland 2012

Fig. 2 (p.100) Section on the old Tremola Road in Ticino Switzerland with the Gotthard Motorway tunnel below, 2014

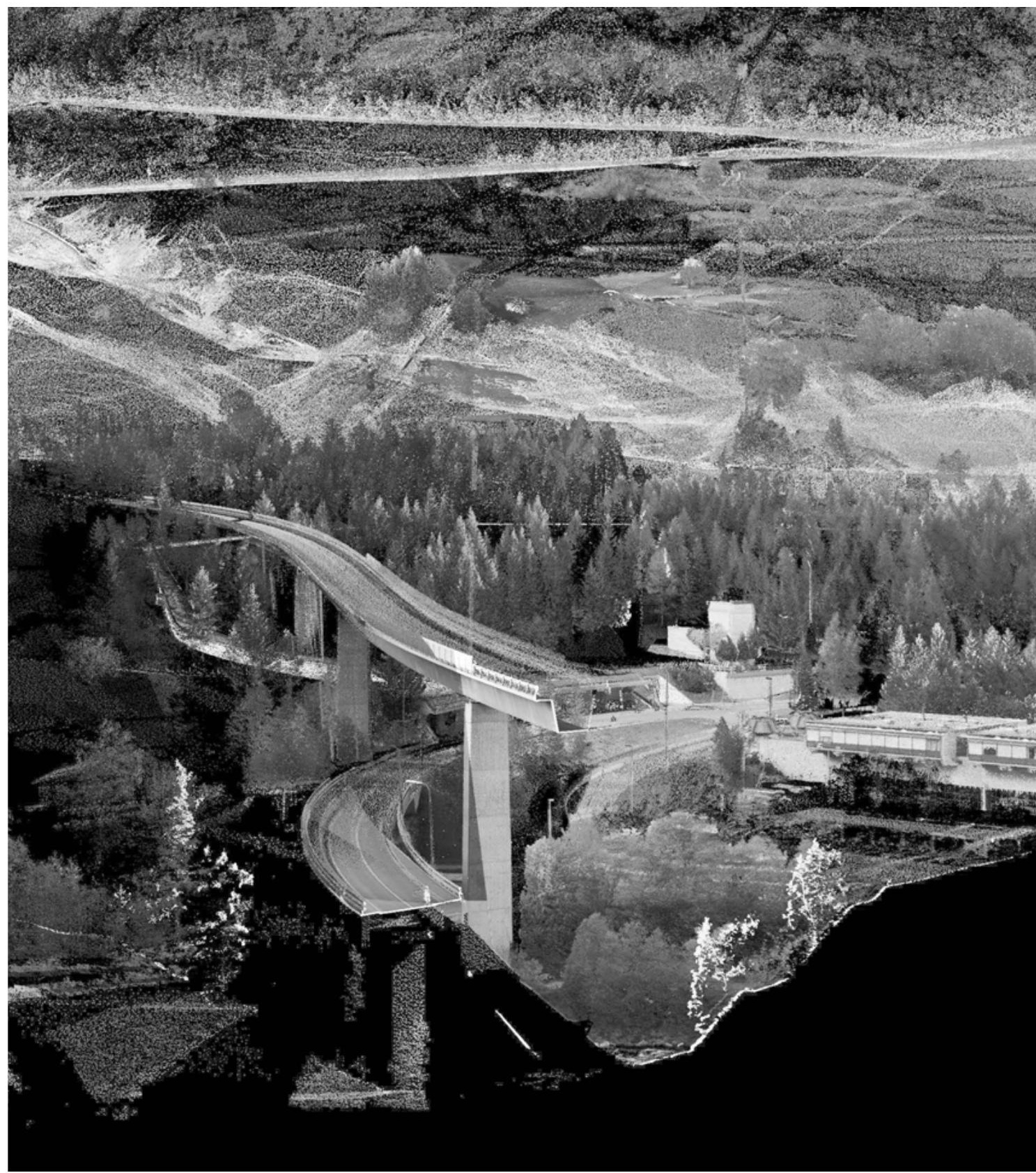




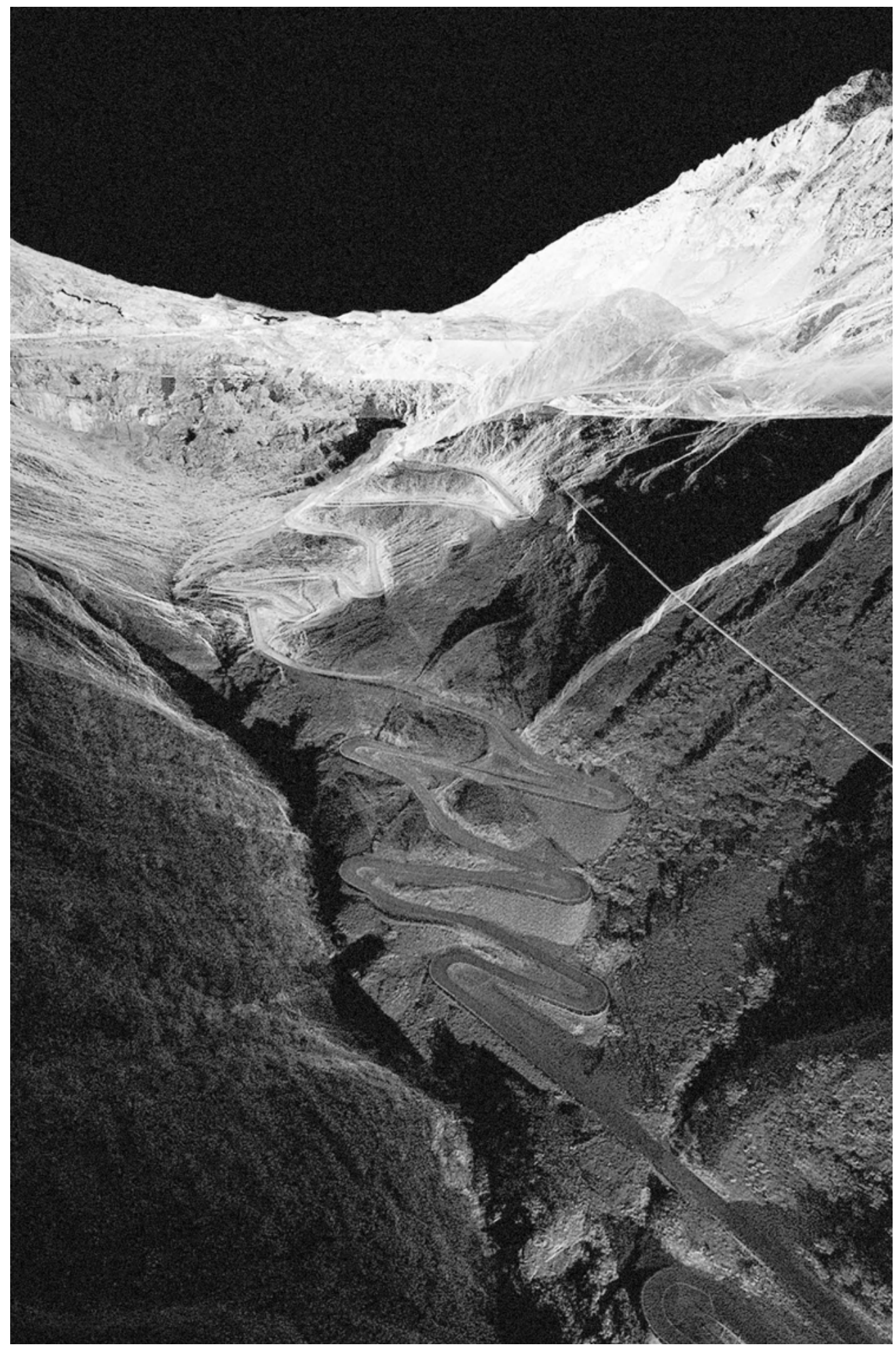


enabled one to see through to the tunnel infrastructures lying deep below the surface at one glance. Although the Gotthard project still only serves a general informational purpose, it has permitted my team to assemble underground elements such as the new Gotthard Base Tunnel (currently the longest tunnel in the world), as well as the older 19th century spiral railway tunnels with a high degree of precision. This modelling feat shows how cloudist technology can serve the extraordinary complexity of the Swiss alpine context, by showing and demystifying an extremely complex terrain simply, beautifully and poetically.

It would, however, be mistaken to consider that the cloudist method applies only to large scale environmental and urban projects, and that the scale of the intimate garden escapes, somehow, from the reach of this technique. A joint research and teaching programme on Japanese gardens between ETH and the Kyoto Institute of Technology (KIT) over the past four years has yielded results of extraordinary precision and delight, combining point clouds with geo-located sound samplings. ${ }^{8}$ One still wonders at the extraordinary versatility of the work done in these short one-week workshops, where the KIT students encapsulate all the wonder, culture and mystery of a traditional Japanese garden in the most exquisite detail. The sound samples, which are located in the model precisely where they were taken, enhance a sense of space and time in the model of the garden. ${ }^{9}$ This acoustic dimension adds a sensory layer to a point cloud model, and it also revives decades of dormant research in landscape acoustics. There is definitely a new aesthetic arising from the cloudist experiments that have been listed. Their digital form and appearance will depend on the task and process at hand. A broad range of projects, from the most engineered and technical to the most artistic and poetic, is opening an array of new possibilities in our discipline. Cloudism is the new horizon that will bring much needed changes to the design, analysis and production of landscape architecture. I am convinced that it is here to stay. Far from being some fickle flash in the pan, it is actually something fundamentally new that is going to contribute significantly to the field of landscape architecture. We just have to learn how to be more creative and grow with it in the decade to come.

Extract from Christophe Girot's "Cloudism: Towards a new culture of making landscapes" in Routledge Research Companion to Landscape Architecture (Routledge 2018), edited by Ellen Braae and Henriette Steiner.
1 Jane Hutton, Material Culture, Landscript 5, Jovis, Berlin 2017.

2 The Sweepnik used a laser with a set of movable mirrors and became the first known laser scanning device on the research market.

3 The Riegl company patented its first laser scanner in the early 1970 s, followed by Cyra Technologies in the early 1990s which was then bought by the Leica company in 2001.

$4 \quad$ Marc Levoy and Turner Whitted; The Use of Points at Display Primitive, Technical Report 85-022, Computer Science Department, University of North Carolina at Chapel Hill, January, 1985.

5 The point methodology introduced more than ten years ago has become the basis of a series of studio projects and research projects at the chair of landscape architecture of Prof. Girot at ETH Zürich.

6 Marianne Burkhalter, Christian Sumi; Der Gotthard / II Gottardo Landscape Myths and Technology,

Scheidegger \& Spies, Zürich 2016.

$7 \quad$ Gotthard Landscape - The Unexpected View, Collateral Event 14th International Architecture Exhibition, La Biennale Venezia. Curators, Christophe Girot, Christian Sumi.

$8 \quad$ Sampling Kyoto Gardens, Pamphlet 21, Chair of Prof. C. Girot, GTA Publishers ETH Zürich 2017 PP 1-72.

9 Nadine Michèle Schütz; The Acoustic Dimension of Landscape Architecture, doctoral dissertation, Department of Architecture ETH Zürich 2018. 